\title{
Sharp Estimates for Green's Functions of Cone-Type Planar Domains
}

\author{
Mohamed Amine Ben Boubaker ${ }^{1}$ and Mohamed Selmi \\ ${ }^{1}$ Preparatory Institute For Engineering Studies of Nabeul, University of Carthage, Campus Universitaire Merezka, 8000 Nabeul, Tunisia \\ ${ }^{2}$ Faculty of Sciences of Tunis, Department of Mathematics, University of Tunis el Manar, Campus Universitaire, 2092 Tunis, Tunisia
}

Correspondence should be addressed to Mohamed Amine Ben Boubaker; mohamedamine.benboubaker@ipein.rnu.tn

Received 19 October 2015; Revised 3 January 2016; Accepted 11 January 2016

Academic Editor: Okay Celebi

Copyright (C) 2016 M. A. Ben Boubaker and M. Selmi. This is an open access article distributed under the Creative Commons Attribution License, which permits unrestricted use, distribution, and reproduction in any medium, provided the original work is properly cited.

We establish sharp estimates for Green's functions of cone-type planar domains. Our work generalizes all estimates given by Zhao in 1988 and Selmi in 2000. Our principal idea is to use conformal mappings.

\section{Introduction}

We work in the Euclidean space $\mathbb{R}^{2}$. By $G_{\Omega}$, we denote the Green function for the Laplacian in a domain $\Omega$. We write $\delta(x)$ for the distance from $x \in \Omega$ to the Euclidean boundary $\partial \Omega$ of $\Omega$. For two positive functions $f$ and $g$ on a set $\Omega$, we say that $f$ is comparable to $g$ on $\Omega$ and we denote $f \simeq g$, if there exists $A \geq 1$, such that $\forall x \in \Omega,(1 / A) g(x) \leq f(x) \leq A g(x)$. The constant $A$ will be called the constant of comparison. For $a, b \in \mathbb{R}$, we denote $(a \vee b)=\max (a, b)$ and $(a \wedge b)=\min (a, b)$. $D(a, s)$ denote the open disk of center $a$ and radius $s$. For $z \in$ $\mathbb{C}$, we denote $r=|z|$ and $\theta=\arg z$. In [1], Zhao established estimates for the Green function on bounded $C^{2}$ domains $\Omega$ in $\mathbb{R}^{2}$. More precisely, he proved that

$$
G_{\Omega}(x, y) \simeq \ln \left(1+\frac{\delta(x) \delta(y)}{|x-y|^{2}}\right), \quad \text { on } \Omega \times \Omega \text {. }
$$

This result was generalized later by Selmi in [2]. He established inequalities for the Green function of a class of Dinismooth Jordan domains in $\mathbb{R}^{2}$. He proved that if $\Omega$ is a bounded multiply connected Dini-smooth Jordan domain in $\mathbb{R}^{2}$, then inequality (1) holds. In [3], under restricted conditions on $\Omega$, Sweers established an implicit formula for the estimate of the Green function; the estimate is expressed as a function of the first eigenfunction. The aim of the present paper is to give estimates for the Green function in some particular bounded and unbounded nonsmooth domains in
$\mathbb{R}^{2}$. Our work generalizes all estimates given by Zhao in [1], Selmi in [2], and Sweers in [3]. Our principal idea is to use conformal mappings. Note that, by the Riemann Theorem (see [4], pages 133-136 and pages 229-233), if $\Omega$ is a nonempty open simply connected domain in $\mathbb{R}^{2}$, distinct of $\mathbb{R}^{2}$, then there exists a conformal mapping from $\Omega$ onto the unit disk $D$. Consequently, for all $x, y \in \Omega$

$$
\begin{aligned}
G_{\Omega}(x, y) & =G_{D}(\phi(x), \phi(y)) \\
& \simeq \ln \left(1+\frac{\left(1-|\phi(x)|^{2}\right)\left(1-|\phi(y)|^{2}\right)}{|\phi(x)-\phi(y)|^{2}}\right)
\end{aligned}
$$

on $\Omega \times \Omega$.

Thus, to give estimates for the Green function, it is sufficient to give estimates for $|\phi(x)-\phi(y)|$ and $1-|\phi(x)|^{2}$ on $\Omega$. The problem reduces to understanding distortion introduced by $\phi$. The paper is organized as follows. In Section 2, we establish some preliminary results that will be necessary throughout this paper. In Section 3, we give estimates for the Green function in $\left.S_{\alpha}=\{z \in \mathbb{C}:|\theta|<\pi / 2 \alpha\}, \alpha \in\right] 1 / 2,+\infty[\backslash\{1\}$. We put $\Delta_{1}=\{z \in \mathbb{C}: r \geq 0, \theta=\pi / 2 \alpha\}$ and $\Delta_{2}=$ $\{z \in \mathbb{C}: r \geq 0, \theta=-\pi / 2 \alpha\}$. For $z \in S_{\alpha}$, we denote $d_{1}(z)=d\left(z, \Delta_{1}\right)\left(\right.$ resp., $\left.d_{2}(z)=d\left(z, \Delta_{2}\right)\right)$, the distance from $z$ to $\Delta_{1}$ (resp., from $z$ to $\Delta_{2}$ ). If $x, y \in S_{\alpha}$, we define the bracket 
$[x, y]_{\alpha, 0}=|x-y|^{2}+4 d_{1}(x) d_{1}(y)+4 d_{2}(x) d_{2}(y)$. We prove the following results.

Theorem 1. Let $\alpha>1 / 2, \alpha \neq 1$. Then

$$
\begin{aligned}
& G_{S_{\alpha}}(x, y) \simeq \ln (1 \\
& \left.+\left(\frac{|x| \wedge|y|}{|x| \vee|y|}\right)^{\alpha-2} \frac{d_{1}(x) d_{1}(y) d_{2}(x) d_{2}(y)}{|x-y|^{2}[x, y]_{\alpha, 0}}\right) \\
& \quad \ln (1 \\
& \left.+\left(\frac{|x| \wedge|y|}{|x| \vee|y|}\right)^{\alpha-2} \frac{d_{1}(x) d_{1}(y) d_{2}(x) d_{2}(y)}{|x-y|^{2}(|x| \vee|y|)^{2}}\right) \\
&
\end{aligned}
$$

Corollary 2. Let $\alpha>1 / 2, \alpha \neq 1$. Then

$$
\begin{array}{r}
G_{S_{\alpha}}(x, y) \simeq \ln \left(1+\frac{\delta(x) \delta(y)}{|x-y|^{2}}\left(\frac{(|x| \wedge|y|)}{(|x| \vee|y|)}\right)^{\alpha-1}\right) \\
\text { on } S_{\alpha} \times S_{\alpha} .
\end{array}
$$

In Section 4, we give estimates for the Green function in $\left.S_{\alpha}(D)=\{z \in \mathbb{C}: 0<r<1,|\theta|<\pi / 2 \alpha\}, \alpha \in\right] 1 / 2,+\infty[\backslash\{1\}$. We put $\Delta_{0,2 \alpha}=\{z \in \mathbb{C}: 0<r<1, \theta=\pi / 2 \alpha\}$, $\Delta_{0,-2 \alpha}=\{z \in \mathbb{C}: 0<r<1, \theta=-\pi / 2 \alpha\}$, and $C_{1,2 \alpha}=\{z \in \mathbb{C}: r=1,|\theta| \leq \pi / 2 \alpha\}$. For $z \in S_{\alpha}(D)$, we denote $\delta_{1}(z)=d\left(z, \Delta_{0,2 \alpha}\right)$ (resp., $\delta_{2}(z)=d\left(z, \Delta_{0,-2 \alpha}\right)$; resp., $\left.\delta_{3}(z)=d\left(z, C_{1,2 \alpha}\right)\right)$, the distance from $z$ to $\Delta_{0,2 \alpha}$ (resp., from $z$ to $\Delta_{0-2 \alpha}$; resp., from $z$ to $\left.C_{1,2 \alpha}\right)$. For $x, y \in S_{\alpha}(D)$, we define the brackets $[x, y]_{\alpha, 1}=|x-y|^{2}+4 \delta_{1}(x) \delta_{1}(y)+4 \delta_{2}(x) \delta_{2}(y)$, $[x, y]_{\alpha, 2}=|x-y|^{2}+4 \delta_{1}(x) \delta_{1}(y)+\left(1-|x|^{2}\right)\left(1-|y|^{2}\right)$, $[x, y]_{\alpha, 3}=|x-y|^{2}+4 \delta_{2}(x) \delta_{2}(y)+\left(1-|x|^{2}\right)\left(1-|y|^{2}\right)$, and $[x, y]_{\alpha}=(|x| \mathrm{V}|y|)^{2}\left(\left|x-e^{i(\pi / 2 \alpha)}\right| \mathrm{V}\left|y-e^{i(\pi / 2 \alpha)}\right|\right)^{2}\left(\left|x-e^{-i(\pi / 2 \alpha)}\right| \mathrm{V}\right.$ $\left.\left|y-e^{-i(\pi / 2 \alpha)}\right|\right)^{2}$. We prove the following.

Theorem 3. Let $\alpha>1 / 2, \alpha \neq 1$. Then

$$
\begin{array}{r}
G_{S_{\alpha}(D)}(x, y) \simeq \ln (1 \\
\left.+\frac{\delta_{1}(x) \delta_{1}(y) \delta_{2}(x) \delta_{2}(y) \delta_{3}(x) \delta_{3}(y)}{|x-y|^{2}[x, y]_{\alpha, 1}[x, y]_{\alpha, 2}[x, y]_{\alpha, 3}}\left(\frac{(|x| \wedge|y|)}{(|x| \vee|y|)}\right)^{\alpha-2}\right), \\
\text { on } S_{\alpha}(D) \times S_{\alpha}(D), \\
G_{S_{\alpha}(D)}(x, y) \simeq \ln (1 \\
\left.+\frac{\delta_{1}(x) \delta_{1}(y) \delta_{2}(x) \delta_{2}(y) \delta_{3}(x) \delta_{3}(y)}{|x-y|^{2}[x, y]_{\alpha}}\left(\frac{(|x| \wedge|y|)}{(|x| \vee|y|)}\right)^{\alpha-2}\right), \\
\text { on } S_{\alpha}(D) \times S_{\alpha}(D) .
\end{array}
$$

Corollary 4. Let $\alpha>1 / 2, \alpha \neq 1$. Then, for all $x, y \in S_{\alpha}(D)$,

$$
\begin{aligned}
& G_{S_{\alpha}(D)}(x, y) \simeq \ln \left(1+\left(\frac{\left|x-e^{i(\pi / 2 \alpha)}\right| \wedge\left|y-e^{i(\pi / 2 \alpha)}\right|}{\left|x-e^{i(\pi / 2 \alpha)}\right| \vee\left|y-e^{i(\pi / 2 \alpha)}\right|}\right)\right. \\
& \cdot\left(\frac{\left|x-e^{-i(\pi / 2 \alpha)}\right| \wedge\left|y-e^{-i(\pi / 2 \alpha)}\right|}{\left|x-e^{-i(\pi / 2 \alpha)}\right| \vee\left|y-e^{-i(\pi / 2 \alpha)}\right|}\right)\left(\frac{|x| \wedge|y|}{|x| \vee|y|}\right)^{\alpha-1} \\
& \left.\cdot \frac{\delta(x) \delta(y)}{|x-y|^{2}}\right) .
\end{aligned}
$$

In Section 5, we give estimates for the Green function in $D \cap D(a, s)$. To study the intersection of two open disk, let us remark that using translations, rotations, and homotheties we reduce the problem to study the situation of $D$ and $D(a, s)$, where $s \in \mathbb{R}_{*}^{+}, 0<s \leq 1$, and $1-s<a<1+s$. The two boundary circles $C(0,1)$ and $C(a, s)$ intersect nontangentially at $e^{ \pm i \theta_{0}}=a+e^{ \pm i \theta_{1}}$ with $\cos \theta_{0}=\left(1+a^{2}-s^{2}\right) / 2 a$ and $\sin \theta_{0}=\left[s^{2}-(a-1)^{2}\right]\left[(1+a)^{2}-s^{2}\right] / 2 a$. The interior angle $\varphi$ of the intersection is given by $\cos \varphi=\left(1+s^{2}-a^{2}\right) / 2 s$ and the boundary of $D \cap D(a, s)$ is formed by the arcs of circles

$$
\begin{aligned}
& \mathscr{C}_{1}=\left\{z \in \mathbb{C}:|z|=1,|\arg z| \leq \theta_{0}\right\}, \\
& \mathscr{C}_{2}=\left\{z \in \mathbb{C}:|z-a|=s,|\arg z| \geq \theta_{1}\right\} .
\end{aligned}
$$

We denote by $\delta_{i}^{\star}(z)$ the distance of $z \in D \cap D\left(a, r_{0}\right)$ to the boundary $\mathscr{C}_{i}$. For $x, y \in D \cap D(a, s)$, we write

$$
\begin{aligned}
& \Delta(x, y) \\
& =\frac{\delta_{1}^{*}(x) \delta_{1}^{*}(y) \delta_{2}^{*}(x) \delta_{2}^{*}(y)}{|x-y|^{2}\left(\left|x-e^{i \theta_{0}}\right| \vee\left|y-e^{i \theta_{0}}\right|\right)^{2}\left(\left|x-e^{-i \theta_{0}}\right| \vee\left|y-e^{-i \theta_{0}}\right|\right)^{2}}
\end{aligned}
$$

We prove the following.

Theorem 5. For all $x, y \in D \cap D(a, s)$,

$$
\begin{aligned}
& G_{D \cap D(a, s)}(x, y) \simeq \ln (1 \\
& +\left(\frac{\left(\left|x-e^{i \theta_{0}}\right| \wedge\left|y-e^{i \theta_{0}}\right|\right)}{\left(\left|x-e^{i \theta_{0}}\right| \vee\left|y-e^{i \theta_{0}}\right|\right)}\right) \\
& \left.\quad \cdot\left(\frac{\left(\left|x-e^{-i \theta_{0}}\right| \wedge\left|y-e^{-i \theta_{0}}\right|\right)}{\left(\left|x-e^{-i \theta_{0}}\right| \vee\left|y-e^{-i \theta_{0}}\right|\right)}\right)^{\alpha-2} \Delta(x, y)\right),
\end{aligned}
$$

where $\alpha=\pi /\left(\pi+\theta_{0}-\theta_{1}\right)>1$. 
Corollary 6. For all $x, y \in D \cap D(a, s)$,

$$
\begin{aligned}
& G_{D \cap D(a, s)}(x, y) \simeq \ln (1 \\
& +\frac{\delta(x) \delta(y)}{|x-y|^{2}}\left(\frac{\left|x-e^{i \theta_{0}}\right| \wedge\left|y-e^{i \theta_{0}}\right|}{\left|x-e^{i \theta_{0}}\right| \vee\left|y-e^{i \theta_{0}}\right|}\right)^{\alpha-1} \\
& \left.\cdot\left(\frac{\left|x-e^{-i \theta_{0}}\right| \wedge\left|y-e^{-i \theta_{0}}\right|}{\left|x-e^{-i \theta_{0}}\right| \vee\left|y-e^{-i \theta_{0}}\right|}\right)^{\alpha-1}\right) .
\end{aligned}
$$

In Section 6, we give estimates for the Green function in $D \cup D(a, s)$. We reduce the problem as in Section 5. We prove the following.

Theorem 7. For all $x, y \in D \cup D(a, s)$,

$$
\begin{aligned}
& G_{D \cup D(a, s)}(x, y) \simeq \ln (1 \\
& +\left(\frac{\left(\left|x-e^{i \theta_{0}}\right| \wedge\left|y-e^{i \theta_{0}}\right|\right)}{\left(\left|x-e^{i \theta_{0}}\right| \vee\left|y-e^{i \theta_{0}}\right|\right)}\right)^{\beta-1} \\
& \left.\quad \cdot\left(\frac{\left(\left|x-e^{-i \theta_{0}}\right| \wedge\left|y-e^{-i \theta_{0}}\right|\right)}{\left(\left|x-e^{-i \theta_{0}}\right| \vee\left|y-e^{-i \theta_{0}}\right|\right)}\right)^{\beta-1} \frac{\delta(x) \delta(y)}{|x-y|^{2}}\right),
\end{aligned}
$$

where $\beta=\pi /\left(\pi-\theta_{0}+\theta_{1}\right)$.

In Section 7, we give estimates for the Green function in $D \backslash D(a, s)$. We reduce the problem as in Section 5 . We prove the following.

Theorem 8. For all $x, y \in D \backslash D(a, s)$,

$$
\begin{aligned}
& G_{D \backslash D(a, s)}(x, y) \simeq \ln (1 \\
& +\left(\frac{\left(\left|x-e^{i \theta_{0}}\right| \wedge\left|y-e^{i \theta_{0}}\right|\right)}{\left(\left|x-e^{i \theta_{0}}\right| \vee\left|y-e^{i \theta_{0}}\right|\right)}\right)^{\gamma-1} \\
& \left.\quad \cdot\left(\frac{\left(\left|x-e^{-i \theta_{0}}\right| \wedge\left|y-e^{-i \theta_{0}}\right|\right)}{\left(\left|x-e^{-i \theta_{0}}\right| \vee\left|y-e^{-i \theta_{0}}\right|\right)}\right)^{\gamma-1} \frac{\delta(x) \delta(y)}{|x-y|^{2}}\right),
\end{aligned}
$$

where $\gamma=\pi /\left(\theta_{1}-\theta_{0}\right)$.

In Section 8, we give estimates for the Green function in $D_{1}=D \cap\left\{z \in \mathbb{C}: \mathfrak{R} e(z)>\cos \theta_{0}\right\}, 0<\theta_{0}<\pi / 2$. We prove the following.
Theorem 9. For all $x, y \in D_{1}$,

$$
\begin{aligned}
& G_{D_{1}}(x, y) \simeq \ln (1 \\
& +\frac{\delta_{1}^{\prime \prime \prime}(x) \delta_{1}^{\prime \prime \prime}(y) \delta_{2}^{\prime \prime \prime}(x) \delta_{2}^{\prime \prime \prime}(y)}{|x-y|^{2}\left(\left|x-e^{i \theta_{0}}\right| \vee\left|y-e^{i \theta_{0}}\right|\right)^{2}\left(\left|x-e^{-i \theta_{0}}\right| \vee\left|y-e^{-i \theta_{0}}\right|\right)^{2}} \\
& \quad \times\left(\frac{\left|x-e^{i \theta_{0}}\right| \wedge\left|y-e^{i \theta_{0}}\right|}{\left|x-e^{i \theta_{0}}\right| \vee\left|y-e^{i \theta_{0}}\right|}\right)^{\nu-2} \\
& \left.+\left(\frac{\left|x-e^{-i \theta_{0}}\right| \wedge\left|y-e^{-i \theta_{0}}\right|}{\left|x-e^{-i \theta_{0}}\right| \vee\left|y-e^{-i \theta_{0}}\right|}\right)^{\nu-2}\right) \simeq \ln (1 \\
& +\frac{\delta(x) \delta(y)}{|x-y|^{2}}\left(\frac{\left|x-e^{i \theta_{0}}\right| \wedge\left|y-e^{i \theta_{0}}\right|}{\left|x-e^{i \theta_{0}}\right| \vee\left|y-e^{i \theta_{0}}\right|}\right)^{\nu-1} \\
& \left.\quad \cdot\left(\frac{\left|x-e^{-i \theta_{0}}\right| \wedge\left|y-e^{-i \theta_{0}}\right|}{\left|x-e^{-i \theta_{0}}\right| \vee\left|y-e^{-i \theta_{0}}\right|}\right)^{\nu-1}\right)
\end{aligned}
$$

where $v=\pi / \theta_{0} \geq 2, \delta_{1}^{\prime \prime \prime}(x)=1-|x|$, and $\delta_{2}^{\prime \prime \prime}(x)=\mathfrak{R} e(x)-$ $\cos \theta_{0}$.

\section{Preliminaries Results}

In this section, the following notations will be adopted. For $\alpha>1 / 2$ and $0<\varepsilon<1$, we denote

$$
\begin{aligned}
D_{\alpha} & =\left\{z \in \mathbb{C}: 0<r<1,0<\theta<\frac{\pi}{\alpha}\right\}, \\
\Omega_{\alpha, \varepsilon} & =\left\{z \in \mathbb{C}: \varepsilon<r<1,|\theta|<\frac{\pi}{\alpha}\right\}, \\
\Delta_{\varepsilon, \alpha} & =\left\{z \in \mathbb{C}: \varepsilon<r<1, \theta=\frac{\pi}{\alpha}\right\}, \\
\Delta_{\varepsilon,-\alpha} & =\left\{z \in \mathbb{C}: \varepsilon<r<1, \theta=\frac{\pi}{-\alpha}\right\}, \\
C_{\varepsilon, \alpha} & =\left\{z \in \mathbb{C}: r=\varepsilon,|\theta| \leq \frac{\pi}{\alpha}\right\} .
\end{aligned}
$$

Lemma 10. Let $\alpha>1 / 2, \alpha \neq 1$. Then,

$$
\sin \alpha \theta \simeq \sin \theta, \quad \text { on }\left[0, \frac{\pi}{2 \alpha}\right] \text {. }
$$

Proof. It suffices to see that

$$
\varphi_{\alpha}(\theta)= \begin{cases}\frac{\sin \alpha \theta}{\sin \theta} & \text { if } \left.\theta \in] 0, \frac{\pi}{2 \alpha}\right] \\ \alpha & \text { if } \theta=0\end{cases}
$$

is continuous and does not vanish on $[0, \pi / 2 \alpha]$.

Lemma 11. Let $\alpha>1$. Then

$$
\left|1-z^{\alpha}\right| \simeq|1-z|, \quad \text { on } S_{\alpha / 2}(D)
$$


Proof. Let $\varepsilon<1 / 4$. The function

$$
f_{\alpha}(z)= \begin{cases}\frac{1-z^{\alpha}}{1-z} & \text { if } z \neq 1 \\ \alpha & \text { if } z=1\end{cases}
$$

is holomorphic on $\Omega_{\alpha, \varepsilon}$ and continuous and does not vanish on $\overline{\Omega_{\alpha, \varepsilon}}$. Then, by maximum and minimum principles, it is sufficient to control $f_{\alpha}$ on $\partial \Omega_{\alpha, \varepsilon}$. We have to discuss three cases.

Case 1. If $z \in C_{1, \alpha}$, then

$$
\left|\frac{1-z^{\alpha}}{1-z}\right|^{2}=\left(\frac{\sin (\alpha(\theta / 2))}{\sin (\theta / 2)}\right)^{2} \text {. }
$$

The result follows from Lemma 10.

Case 2. If $z \in C_{\varepsilon, \alpha}$, then

$$
\frac{3}{8} \leq\left|\frac{1-z^{\alpha}}{1-z}\right| \leq \frac{8}{3}
$$

Case 3. If $z \in \Delta_{\varepsilon, \alpha} \cup \Delta_{\varepsilon,-\alpha}$, then

$$
\begin{aligned}
\left|\frac{1-z^{\alpha}}{1-z}\right|^{2} & =\frac{\left(1+r^{\alpha}\right)^{2}}{1+r^{2}-2 r \cos (\pi / \alpha)} \\
& \leq \frac{\left(1+r^{\alpha}\right)^{2}}{(1-r)^{2}+4 r \sin ^{2}(\pi / 2 \alpha)}
\end{aligned}
$$

So, we get

$$
\frac{1}{4} \leq\left|\frac{1-z^{\alpha}}{1-z}\right|^{2} \leq \frac{20}{\sin ^{2}(\pi / 2 \alpha)}
$$

Since the constants are independent of $\varepsilon$, we obtain, for all $z \in S_{\alpha / 2}(D)$,

$$
\left|1-z^{\alpha}\right| \simeq|1-z|
$$

Lemma 12. Let $\alpha \in] 1 / 2,1[$. Then

$$
\left|1-z^{\alpha}\right| \simeq|1-z|, \quad \text { on } D_{\alpha} \text {. }
$$

Proof. The proof is similar to that of Lemma 11. Let $\varepsilon<1 / 4$. The function

$$
g_{\alpha}(z)= \begin{cases}\frac{z^{\alpha}+i}{z-e^{-i(\pi / 2 \alpha)}} & \text { if } z \neq e^{-i(\pi / 2 \alpha)}, \\ \alpha e^{-i(\pi(\alpha-1) / 2 \alpha)} & \text { if } z=e^{-i(\pi / 2 \alpha)}\end{cases}
$$

is holomorphic on $\Omega_{2 \alpha, \varepsilon}$ and continuous and does not vanish on $\overline{\Omega_{2 \alpha, \varepsilon}}$. By maximum and minimum principles, it is sufficient to control $g_{\alpha}$ on $\partial \Omega_{2 \alpha, \varepsilon}$. We have to discuss four cases.

Case 1. If $z \in C_{1,2 \alpha}$, then

$$
\left|g_{\alpha}(z)\right|^{2}=\frac{1-\sin \alpha \theta}{1-\cos (\theta+\pi / 2 \alpha)}=\left(\frac{\sin \alpha \nu}{\sin \nu}\right)^{2},
$$

where $v=(\theta+\pi / 2 \alpha) / 2 \in[0, \pi / 2 \alpha]$. The result follows from Lemma 10 .

Case 2. If $z \in C_{\varepsilon, 2 \alpha}$, then

$$
\frac{1}{4}<\frac{1-\varepsilon^{\alpha}}{1+\varepsilon} \leq\left|g_{\alpha}(z)\right| \leq \frac{1+\varepsilon^{\alpha}}{1-\varepsilon}<\frac{8}{3} .
$$

Case 3. If $z \in \Delta_{\varepsilon, 2 \alpha}$, then

$$
\frac{1}{4} \leq\left|g_{\alpha}(z)\right|^{2}=\frac{\left(1+r^{\alpha}\right)^{2}}{r^{2}+1-2 r \cos (\pi / \alpha)} \leq \frac{20}{\sin ^{2}(\pi / 2 \alpha)}
$$

Case 4. If $z \in \Delta_{\varepsilon,-2 \alpha}$, then

$$
\frac{1}{2} \leq\left|g_{\alpha}(z)\right|=\frac{1-r^{\alpha}}{1-r}=\frac{\int_{r}^{1} \alpha t^{\alpha-1} d t}{\int_{r}^{1} d t} \leq 1+\alpha
$$

As the constants are independent of $\varepsilon$, we obtain for all $z \in$ $S_{\alpha}(D)$

$$
\left|z^{\alpha}+i\right| \simeq\left|z-e^{-i(\pi / 2 \alpha)}\right|
$$

Now let $z \in D_{\alpha}$; then $e^{-i(\pi / 2 \alpha)} z \in S_{\alpha}(D)$. By using the previous relation, we get, for all $z \in D_{\alpha}$,

$$
\left|z^{\alpha}-1\right| \simeq|z-1|
$$

By using the conjugate expression in the last lemma, we obtain the following.

Lemma 13. Let $\alpha \in] 1 / 2,1[$. Then

$$
\left|1-z^{\alpha}\right| \simeq|1-z|, \quad \text { on } D \backslash D_{\alpha /(2 \alpha-1)} \text {. }
$$

Lemma 14. Let $\alpha>1 / 2, \alpha \neq 1$. Then,

$$
\left|x^{\alpha}-y^{\alpha}\right| \simeq|x-y|(|x| \vee|y|)^{\alpha-1}, \quad \text { on } S_{\alpha} \times S_{\alpha}
$$

Proof. We have to discuss two cases.

Case 1. If $\alpha>1$, assume that $|x|>|y|$ and put $z=y / x$. Then, $|z|<1$ and $|\arg z| \leq \pi / \alpha$. Since

$$
\left|\frac{x^{\alpha}-y^{\alpha}}{x-y}\right|=\left|x^{\alpha-1}\right|\left|\frac{1-z^{\alpha}}{1-z}\right|,
$$

then the result follows by Lemma 11 . 
Case 2. If $\alpha \in] 1 / 2,1[$, assume that $|x|>|y|$ and put $z=y / x$. Then, $|z|<1$ and $\arg z \in[0, \pi / \alpha]$, or $\arg z \in[\pi /(\alpha /(2 \alpha-$ $1)), 2 \pi]$. Since

$$
\left|\frac{x^{\alpha}-y^{\alpha}}{x-y}\right|=\left|x^{\alpha-1}\right|\left|\frac{1-z^{\alpha}}{1-z}\right|
$$

then the result follows by Lemmas 12 and 13 .
Lemma 15. Let $\alpha>1 / 2, \alpha \neq 1$. Then

$$
\begin{array}{r}
\cos \alpha \theta \simeq\left[\sin ^{2}\left(\frac{\pi}{2 \alpha}-\theta\right)+\left(0 \wedge \cos \left(\frac{\pi}{2 \alpha}-\theta\right)\right)^{2}\right]^{1 / 2} \\
\cdot\left[\sin ^{2}\left(\frac{\pi}{2 \alpha}+\theta\right)+\left(0 \wedge \cos \left(\frac{\pi}{2 \alpha}+\theta\right)\right)^{2}\right]^{1 / 2}, \\
\text { on }\left[-\frac{\pi}{2 \alpha}, \frac{\pi}{2 \alpha}\right] .
\end{array}
$$

Proof. The function

$$
f_{\alpha}(\theta)=\frac{\cos \alpha \theta}{\left[\sin ^{2}(\pi / 2 \alpha-\theta)+(0 \wedge \cos (\pi / 2 \alpha-\theta))^{2}\right]^{1 / 2}\left[\sin ^{2}(\pi / 2 \alpha+\theta)+(0 \wedge \cos (\pi / 2 \alpha+\theta))^{2}\right]^{1 / 2}}
$$

is continuous and even and does not vanish on ] $-\pi / 2 \alpha$, $\pi / 2 \alpha$. To study $f_{\alpha}$ on a neighborhood of $\pi / 2 \alpha$, we put $\nu=$ $\pi / 2 \alpha-\theta \in[0, \varepsilon[\subset[0, \pi / 2]$. Hence

$$
\begin{aligned}
& f_{\alpha}(\theta)=f_{\alpha}\left(\frac{\pi}{2 \alpha}-\nu\right) \\
& =\frac{\sin (\alpha \nu)}{\sin (\nu)} \frac{1}{\sqrt{\sin ^{2}(\pi / \alpha-\nu)+(0 \wedge \cos (\pi / \alpha-\nu))^{2}}}, \\
& \lim _{\nu \rightarrow 0} f_{\alpha}\left(\frac{\pi}{2 \alpha}-\nu\right)= \begin{cases}\alpha & \text { if } \cos \frac{\pi}{\alpha}<0 \\
\frac{\alpha}{\sin (\pi / \alpha)} & \text { if } \cos \frac{\pi}{\alpha} \geq 0 .\end{cases}
\end{aligned}
$$

As $\lim _{\theta \rightarrow \pi / 2} f_{\alpha}(\theta) \neq 0$, the function $f_{\alpha}$ can be extended by continuity on $[-\pi / 2 \alpha, \pi / 2 \alpha]$ and the compacity argument finishes the proof.

\section{Estimates for the Green Function on the Sector $S_{\alpha}$}

It is clear that

$$
\begin{aligned}
& d_{1}(z)=r \sqrt{\sin ^{2}\left(\frac{\pi}{2 \alpha}-\theta\right)+\left(0 \wedge \cos \left(\frac{\pi}{2 \alpha}-\theta\right)\right)^{2}}, \\
& d_{2}(z)=r \sqrt{\sin ^{2}\left(\frac{\pi}{2 \alpha}+\theta\right)+\left(0 \wedge \cos \left(\frac{\pi}{2 \alpha}+\theta\right)\right)^{2}} .
\end{aligned}
$$

Lemma 16. Let $\alpha>1 / 2, \alpha \neq 1$, Then

$$
[x, y]_{\alpha, 0} \simeq(|x| \vee|y|)^{2}, \quad \text { on } S_{\alpha} \text {. }
$$

Proof. First, we remark that

$$
\begin{aligned}
{[x, y]_{\alpha, 0} } & \leq|x-y|^{2}+8|x||y| \\
& \leq|x|^{2}+|y|^{2}+10|x||y| \leq 6\left(|x|^{2}+|y|^{2}\right) \\
& \leq 12\left(|x|^{2} \vee|y|^{2}\right)
\end{aligned}
$$

On the other hand, the bracket $[x, y]_{\alpha, 0}$ satisfies

$$
\begin{gathered}
{[x, y]_{\alpha, 0} \geq|x|^{2}+|y|^{2}+2|x||y|} \\
\cdot\left[-\cos ^{2} \frac{\pi}{2 \alpha} \cos (\arg x) \cos (\arg y)\right. \\
\left.-\sin ^{2} \frac{\pi}{2 \alpha} \sin (\arg x) \sin (\arg y)\right] .
\end{gathered}
$$

Let

$$
\begin{aligned}
& K_{\alpha}:\left[-\frac{\pi}{2 \alpha}, \frac{\pi}{2 \alpha}\right] \times\left[-\frac{\pi}{2 \alpha}, \frac{\pi}{2 \alpha}\right] \longrightarrow \mathbb{R} \\
& (\theta, \varphi) \longmapsto-\cos ^{2} \frac{\pi}{2 \alpha} \cos \theta \cos \varphi-\sin ^{2} \frac{\pi}{2 \alpha} \sin \theta \sin \varphi .
\end{aligned}
$$

Obviously, $K_{\alpha}$ is continuous and $\left|K_{\alpha}\right| \leq 1$. Assume that $K_{\alpha}(\theta, \varphi)=1$; we see that $\cos (\pi / 2 \alpha) \neq 0$ and $\sin (\pi / 2 \alpha) \neq 0$. In consequence, $\cos ^{2}(\pi / 2 \alpha)(1+\cos \theta \cos \varphi)+\sin ^{2}(\pi / 2 \alpha)(1+$ $\sin \theta \sin \varphi)=0$. This implies the contradiction $(1+$ $\cos \theta \cos \varphi=0$ and $1+\sin \theta \sin \varphi=0)$. Also, $K_{\alpha}(\theta, \varphi)=$ -1 implies the contradiction $(1-\cos \theta \cos \varphi=0$ and $1-$ $\sin \theta \sin \varphi=0)$. By compacity argument, $\min \left(K_{\alpha}(\theta, \varphi)\right)=k_{\alpha}$ with $-1<k_{\alpha}<1$. This leads us to

$$
\begin{aligned}
|x|^{2} & +|y|^{2}+2|x||y| K_{\alpha}(\arg x, \arg y) \\
& \geq|x|^{2}+|y|^{2}+2|x||y| k_{\alpha} \\
& \geq\left(1-k_{\alpha}^{2}\right)\left(|x|^{2} \vee|y|^{2}\right)^{2}, \quad \text { with } 1-k_{\alpha}^{2}>0 .
\end{aligned}
$$

Lemma 17. Let $\alpha>1 / 2, \alpha \neq 1$. Then

$$
\left(d_{1}(z) \vee d_{2}(z)\right) \simeq|z| \quad \text { on } S_{\alpha} \text {. }
$$

Proof. It is clear that $d_{i}(z) \leq|z|$ for all $z \in S_{\alpha}, i=1,2$. Moreover

$$
\begin{aligned}
& d_{1}(z)+d_{2}(z) \\
& \quad \geq\left(\left|\sin \left(\frac{\pi}{2 \alpha}-\theta\right)\right|+\left|\sin \left(\frac{\pi}{2 \alpha}+\theta\right)\right|\right)|z| .
\end{aligned}
$$


The function

$$
\begin{aligned}
h:\left[-\frac{\pi}{2 \alpha}, \frac{\pi}{2 \alpha}\right] & \longrightarrow \mathbb{R} \\
\sigma & \longmapsto h(\sigma) \\
& =\left|\sin \left(\frac{\pi}{2 \alpha}-\sigma\right)\right|+\left|\sin \left(\frac{\pi}{2 \alpha}+\sigma\right)\right|
\end{aligned}
$$

is continuous and even and does not vanish. In fact $\sin (\pi / 2 \alpha-$ $\sigma)=\sin (\pi / 2 \alpha+\sigma)=0$ allows us to conclude that $\pi / \alpha=n \pi$ with $n \in \mathbb{N}$, where the contradiction is clear enough. Then $\inf h>0$.

For $\alpha>1 / 2, \alpha \neq 1, z \in S_{\alpha}$, we denote by $\delta(z)=\left(d_{1}(z) \wedge\right.$ $\left.d_{2}(z)\right)$ the distance of $z$ to the boundary of $S_{\alpha}$.

Proof of Theorem 1. If $\alpha=1$, then $\forall x, y \in S_{1}$ :

$$
\begin{aligned}
G_{S_{1}}(x, y) & =\ln \left|\frac{x+\bar{y}}{x-y}\right| \\
& =\frac{1}{2} \ln \left(1+\frac{4 \Re e(x) \Re e(y)}{|x-y|^{2}}\right) .
\end{aligned}
$$

As, for $\alpha \neq 1$, the function $z \mapsto z^{\alpha}$ is a conformal mapping from $S_{\alpha}$ onto $S_{1}$, then we have

$$
\begin{array}{r}
G_{S_{\alpha}}(x, y) \\
=\frac{1}{2} \ln \left(1+\frac{|x|^{\alpha} \cos (\alpha \arg x)|y|^{\alpha} \cos (\alpha \arg y)}{\left|x^{\alpha}-y^{\alpha}\right|}\right), \\
\forall x, y \in S_{\alpha} .
\end{array}
$$

By Lemma 15, we remark that $|x|^{2} \cos (\alpha \arg x) \simeq d_{1}(x) d_{2}(x)$ and $|y|^{2} \cos (\alpha \arg y) \simeq d_{1}(y) d_{2}(y)$. Then,

$$
\begin{aligned}
& |x|^{\alpha} \cos (\alpha \arg x)|y|^{\alpha} \cos (\alpha \arg y) \\
& \quad \simeq d_{1}(x) d_{1}(y) d_{2}(x) d_{2}(y)|x|^{\alpha-2}|y|^{\alpha-2} .
\end{aligned}
$$

On the other hand, by Lemmas 11, 14, and 16, we get

$$
\begin{aligned}
\left|x^{\alpha}-y^{\alpha}\right|^{2} & \simeq|x-y|^{2}(|x| \vee|y|)^{2 \alpha-2} \\
& \simeq|x-y|^{2}[x, y]_{\alpha, 0}(|x| \vee|y|)^{2 \alpha-4}
\end{aligned}
$$

So, we obtain

$$
\begin{aligned}
& G_{S_{\alpha}}(x, y) \simeq \ln (1 \\
& \left.+\frac{d_{1}(x) d_{1}(y) d_{2}(x) d_{2}(y)}{|x-y|^{2}[x, y]_{\alpha, 0}}\left(\frac{(|x| \wedge|y|)}{(|x| \vee|y|)}\right)^{\alpha-2}\right) \\
& \simeq \ln (1 \\
& \left.+\frac{d_{1}(x) d_{1}(y) d_{2}(x) d_{2}(y)}{|x-y|^{2}\left(|x|^{2} \vee|y|^{2}\right)}\left(\frac{(|x| \wedge|y|)}{(|x| \vee|y|)}\right)^{\alpha-2}\right) .
\end{aligned}
$$

Now we present two proofs of Corollary 2.

First Proof of Corollary 2. By using Lemma 17, we get, for all $z \in S_{\alpha}$,

$$
\begin{aligned}
\delta(z) & =\left(d_{1}(z) \wedge d_{2}(z)\right) \simeq \frac{d_{1}(z) d_{2}(z)}{d_{1}(z)+d_{2}(z)} \\
& \simeq \frac{d_{1}(z) d_{2}(z)}{|z|} .
\end{aligned}
$$

By substituting the previous expression in relation (3), we get

$$
\begin{aligned}
G_{S_{\alpha}}(x, y) & \simeq \ln \left(1+\frac{d_{1}(x) d_{1}(y) d_{2}(x) d_{2}(y)}{|x-y|^{2}(|x| \vee|y|)^{2}}\left(\frac{(|x| \wedge|y|)}{(|x| \vee|y|)}\right)^{\alpha-2}\right) \\
& \simeq \ln \left(1+\frac{\left(d_{1}(x) d_{2}(x) /|x|\right)\left(d_{1}(y) d_{2}(y) /|y|\right)|x||y|}{|x-y|^{2}(|x| \vee|y|)^{2}}\left(\frac{(|x| \wedge|y|)}{(|x| \vee|y|)}\right)^{\alpha-2}\right) \\
& \simeq \ln \left(1+\frac{\delta(x) \delta(y)|x||y|}{|x-y|^{2}(|x| \vee|y|)^{2}}\left(\frac{(|x| \wedge|y|)}{(|x| \vee|y|)}\right)^{\alpha-2}\right) \simeq \ln \left(1+\frac{\delta(x) \delta(y)}{|x-y|^{2}}\left(\frac{(|x| \wedge|y|)}{(|x| \vee|y|)}\right)^{\alpha-1}\right), \quad \text { on } S_{\alpha} .
\end{aligned}
$$

Second Proof of Corollary 2. As, for $\alpha \neq 1$, the conformal mapping $\phi_{\alpha}(z)=\left(z^{\alpha}-1\right) /\left(z^{\alpha}+1\right)$, from $S_{\alpha}$ onto $D, 0$ is the only singular point of order $\alpha-1$ and $\phi_{\alpha}(z) \simeq \phi_{\alpha}(0)+2 z^{\alpha}$ in a neighborhood of 0 . Moreover for $x, y \in S_{\alpha}$

$$
\begin{aligned}
& G_{S_{\alpha}}(x, y)=G_{D}\left(\phi_{\alpha}(x), \phi_{\alpha}(y)\right) \\
& \quad \simeq \ln \left(1+\frac{\left(1-\left|\phi_{\alpha}(x)\right|^{2}\right)\left(1-\left|\phi_{\alpha}(y)\right|^{2}\right)}{\left|\phi_{\alpha}(x)-\phi_{\alpha}(y)\right|^{2}}\right) .
\end{aligned}
$$


We observe that

$$
\begin{aligned}
\left|\phi_{\alpha}(x)-\phi_{\alpha}(y)\right|^{2} & =\frac{4\left|x^{\alpha}-y^{\alpha}\right|^{2}}{\left|\left(x^{\alpha}+1\right)\left(y^{\alpha}+1\right)\right|^{2}} \\
& \simeq 4|x-y|^{2} \frac{(|x| \vee|y|)^{2 \alpha-2}}{\left|\left(x^{\alpha}+1\right)\right|^{2}\left|\left(y^{\alpha}+1\right)\right|^{2}}
\end{aligned}
$$

and for all $z \in S_{\alpha}$

$$
\begin{aligned}
1-\left|\phi_{\alpha}(z)\right|^{2} & =\frac{4|z|^{\alpha-1}|z| \cos (\alpha \arg z)}{\left|\left(z^{\alpha}+1\right)\right|^{2}} \\
& \simeq \delta(z)\left|\phi_{\alpha}^{\prime}(z)\right| .
\end{aligned}
$$

Consequently,

$$
\begin{aligned}
& \left|\phi_{\alpha}(x)-\phi_{\alpha}(y)\right|^{2} \\
& \quad \simeq 4|x-y|^{2}\left|\phi_{\alpha}^{\prime}(x)\right|\left|\phi_{\alpha}^{\prime}(y)\right| \frac{(|x| \vee|y|)^{2 \alpha-2}}{|x|^{\alpha-1}|y|^{\alpha-1}}
\end{aligned}
$$

This implies that for all $x, y \in S_{\alpha}$

$$
G_{S_{\alpha}}(x, y) \simeq \ln \left(1+\frac{\delta(x) \delta(y)}{|x-y|^{2}}\left(\frac{|x| \wedge|y|}{|x| \vee|y|}\right)^{\alpha-1}\right) .
$$

\section{Estimates of the Green Function on $S_{\alpha}(D)$}

It is clear that

$$
\begin{aligned}
& \delta_{1}(z)=r \sqrt{\sin ^{2}\left(\frac{\pi}{2 \alpha}-\theta\right)+\left(0 \wedge \cos \left(\frac{\pi}{2 \alpha}-\theta\right)\right)^{2}}, \\
& \delta_{2}(z)=r \sqrt{\sin ^{2}\left(\frac{\pi}{2 \alpha}+\theta\right)+\left(0 \wedge \cos \left(\frac{\pi}{2 \alpha}+\theta\right)\right)^{2}}
\end{aligned}
$$

and $\delta_{3}(z)=1-r \simeq 1-r^{2}$. We see that $[x, y]_{\alpha, 2}>$ 0 on $\overline{S_{\alpha}(D) \times S_{\alpha}(D)} \backslash\left(e^{i(\pi / 2 \alpha)}, e^{i(\pi / 2 \alpha)}\right)$ and $[x, y]_{\alpha, 3}>0$ on $\overline{S_{\alpha}(D) \times S_{\alpha}(D)} \backslash\left(e^{-i(\pi / 2 \alpha)}, e^{-i(\pi / 2 \alpha)}\right)$. In the same way in Lemmas 11, 12, and 13, we prove the following lemma.

Lemma 18. Consider

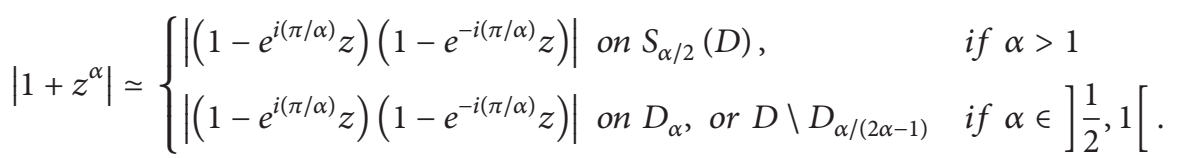

By using the last Lemma, we get the following.

Lemma 19. Let $\alpha>1 / 2, \alpha \neq 1$. Then

$$
\begin{array}{r}
\left|1+x^{\alpha} y^{\alpha}\right| \simeq\left|\left(1-e^{i(\pi / \alpha)} x y\right)\left(1-e^{-i(\pi / \alpha)} x y\right)\right| \\
\quad \text { on } S_{\alpha}(D) \times S_{\alpha}(D) .
\end{array}
$$

Lemma 20. Let $\alpha>1 / 2, \alpha \neq 1$. Then

$$
\begin{aligned}
\left|1-e^{-i(\pi / \alpha)} x y\right|^{2} & \simeq[x, y]_{\alpha, 2}, \\
\left|1-e^{i(\pi / \alpha)} x y\right|^{2} & \simeq[x, y]_{\alpha, 3} \\
& \text { on } S_{\alpha}(D) \times S_{\alpha}(D) .
\end{aligned}
$$

Proof. By symmetry, it is sufficient to prove the result for $[x, y]_{\alpha, 2}$. We see that, for all $\alpha>1 / 2$ and $x, y \in S_{\alpha}(D)$,

$$
\left|1-e^{-i(\pi / \alpha)} x y\right|^{2} \leq[x, y]_{\alpha, 2} .
$$

Moreover, if $\alpha \geq 2$,

$$
[x, y]_{\alpha, 2}=\left|1-e^{-i(\pi / \alpha)} x y\right|^{2} .
$$

If $1<\alpha<2$, then for $z \in S_{\alpha}(D)$

$$
\delta_{1}(z) \leq \frac{r \sin (\pi / 2 \alpha-\theta)}{\cos (\pi / 2 \alpha)}
$$

Since $\sin (\pi / 2 \alpha-\theta)$ is positive and $\cos (\pi / 2 \alpha-\theta)<0$ for $\pi / 2<\pi / 2 \alpha-\theta<\pi / 2+\pi / 2 \alpha<\pi$, then $\sin (\pi / 2 \alpha-\theta) \geq$ $\sin (\pi / 2+\pi / 2 \alpha)=\cos (\pi / 2 \alpha)>0$. This implies for $x, y \in$ $S_{\alpha}(D)$ that

$$
[x, y]_{\alpha, 2} \leq \frac{1}{\cos ^{2}(\pi / 2 \alpha)}\left|1-e^{-i(\pi / \alpha)} x y\right|^{2} .
$$

Finally, for $1 / 2<\alpha<1$, we consider the function

$$
f_{\alpha}(x, y)=\frac{[x, y]_{\alpha, 2}}{\left|1-e^{-i(\pi / \alpha)} x y\right|^{2}}
$$

$$
x, y \in \overline{S_{\alpha}(D) \times S_{\alpha}(D)} \text {. }
$$

$f_{\alpha}$ is continuous and does not vanish in $\overline{S_{\alpha}(D) \times S_{\alpha}(D)}$ । $\left(e^{i(\pi / 2 \alpha)}, e^{i(\pi / 2 \alpha)}\right)$. We will prove that $f_{\alpha}$ is continuous at $\left(e^{i(\pi / 2 \alpha)}, e^{i(\pi / 2 \alpha)}\right)$ and does not vanish. Let $\varepsilon>0$. Every $z \in$ $D\left(e^{i(\pi / 2 \alpha)}, \varepsilon\right) \cap \overline{S_{\alpha}(D)}$ can be written as $z=e^{i(\pi / 2 \alpha)}+t e^{i \theta}$ with $0 \leq t \leq \varepsilon$. Besides, $z \in D\left(e^{i(\pi / 2 \alpha)}, \varepsilon\right) \cap \overline{S_{\alpha}(D)}$ if and only if $\cos (\pi / 2 \alpha-\theta)<-t / 2 \epsilon]-\varepsilon / 2,0]$. This implies that $(\pi / 2 \alpha-\theta) \in[\pi / 2, \arccos (-\varepsilon / 2)[$, and $\operatorname{sos} \sin (\pi / 2 \alpha-\theta) \geq 0$ and $\delta_{1}(z)=\left|\Im m\left(z e^{-i(\pi / \alpha)}\right)\right|=|t \sin (\pi / 2 \alpha-t)|= \pm t \sin (\pi / 2 \alpha-\theta)$. This proves that $\left|1-e^{-i(\pi / \alpha)} x y\right|^{2}=[x, y]_{\alpha, 2}$ for $x, y \in$ $D\left(e^{i(\pi / 2 \alpha)}, \varepsilon\right) \cap \overline{S_{\alpha}(D)}$ and $f_{\alpha}(x, y)=1$ in a neighborhood of $\left.\left(e^{i(\pi / 2 \alpha)}\right), e^{i(\pi / 2 \alpha)}\right)$. This enables us to extend $f_{\alpha}$ continuously on $\left(e^{i(\pi / 2 \alpha)}, e^{i(\pi / 2 \alpha)}\right)$ by 1 . The compacity argument ensures the proof. 
Lemma 21. Let $\alpha>1 / 2, \alpha \neq 1$. Then

$$
[x, y]_{\alpha, 2}[x, y]_{\alpha, 3} \simeq\left|1+x^{\alpha} y^{\alpha}\right|^{2}, \quad \text { on } S_{\alpha}(D) \text {. }
$$

Proof. The proof follows from Lemmas 19 and 20.

Proposition 22. Let $\alpha>1 / 2$. Then

$$
\begin{aligned}
\left|1+x^{\alpha} y^{\alpha}\right| \simeq & \left(\left|x-e^{i(\pi / 2 \alpha)}\right| \vee\left|y-e^{i(\pi / 2 \alpha)}\right|\right) \\
\cdot\left(\left|x-e^{-i(\pi / 2 \alpha)}\right| \vee\left|y-e^{-i(\pi / 2 \alpha)}\right|\right) & \\
& \text { on } S_{\alpha}(D) \times S_{\alpha}(D) .
\end{aligned}
$$

Proof. We equip $\mathbb{C} \times \mathbb{C}$ with the norm $|(x, y)|=\sqrt{|x|^{2}+|y|^{2}}$, $(x, y) \in \mathbb{C} \times \mathbb{C}$. We have

$$
(|x| \vee|y|) \leq|(x, y)| \leq \sqrt{2}(|x| \vee|y|) .
$$

Let $\alpha>1 / 2$. The function

$$
\begin{aligned}
\left.\left.f_{\alpha}:(\mathbb{C} \backslash]-\infty, 0\right]\right)^{2} & \longrightarrow \mathbb{C} \\
(x, y) & \longmapsto x^{\alpha} y^{\alpha}
\end{aligned}
$$

is continuous and differentiable in two points $\left(e^{i(\pi / 2 \alpha)}\right.$, $\left.e^{i(\pi / 2 \alpha)}\right),\left(e^{-i(\pi / 2 \alpha)}, e^{-i(\pi / 2 \alpha)}\right)$ and

$$
\begin{aligned}
& \lim _{(x, y) \rightarrow\left(e^{i(\pi / 2 \alpha)}, e^{i(\pi / 2 \alpha)}\right)} \frac{\left|f_{\alpha}(x, y)-f_{\alpha}\left(e^{i(\pi / 2 \alpha)}, e^{i(\pi / 2 \alpha)}\right)\right|}{\left|(x, y)-\left(e^{i(\pi / 2 \alpha)}, e^{i(\pi / 2 \alpha)}\right)\right|} \\
& =\lim _{(x, y) \rightarrow\left(e^{-i(\pi / 2 \alpha)}, e^{-i(\pi / 2 \alpha)}\right)} \frac{\left|f_{\alpha}(x, y)-f_{\alpha}\left(e^{-i(\pi / 2 \alpha)}, e^{-i(\pi / 2 \alpha)}\right)\right|}{\left|(x, y)-\left(e^{-i(\pi / 2 \alpha)}, e^{-i(\pi / 2 \alpha)}\right)\right|} \\
& =\alpha \sqrt{2} .
\end{aligned}
$$

It follows that

$$
\begin{aligned}
& \lim _{(x, y) \rightarrow\left(e^{i(\pi / 2 \alpha)}, e^{i(\pi / 2 \alpha)}\right)} \frac{\left|1+x^{\alpha} y^{\alpha}\right|}{\left|(x, y)-\left(e^{i(\pi / 2 \alpha)}, e^{i(\pi / 2 \alpha)}\right)\right|} \\
& =\lim _{(x, y) \rightarrow\left(e^{-i(\pi / 2 \alpha)}, e^{-i(\pi / 2 \alpha)}\right)} \frac{\left|1+x^{\alpha} y^{\alpha}\right|}{\left|(x, y)-\left(e^{-i(\pi / 2 \alpha)}, e^{-i(\pi / 2 \alpha)}\right)\right|} \\
& =\alpha \sqrt{2} .
\end{aligned}
$$

Hence, the function

$$
\begin{aligned}
& g_{\alpha}(x, y) \\
& =\frac{\left|1+x^{\alpha} y^{\alpha}\right|}{\left|(x, y)-\left(e^{i(\pi / 2 \alpha)}, e^{i(\pi / 2 \alpha)}\right)\right|\left|(x, y)-\left(e^{-i(\pi / 2 \alpha)}, e^{-i(\pi / 2 \alpha)}\right)\right|}
\end{aligned}
$$

defined on $\overline{S_{\alpha}(D) \times S_{\alpha}(D)}$ is continuous, with $g_{\alpha}\left(e^{i(\pi / 2 \alpha)}\right.$, $\left.e^{i(\pi / 2 \alpha)}\right)=g_{\alpha}\left(e^{-i(\pi / 2 \alpha)}, e^{-i(\pi / 2 \alpha)}\right)=\alpha / 2 \sin (\pi / 2 \alpha) \neq 0$. Moreover, the numerator and the denominator of $g_{\alpha}$ do not vanish on $\overline{S_{\alpha}(D) \times S_{\alpha}(D)} \backslash\left(e^{i(\pi / 2 \alpha)}, e^{i(\pi / 2 \alpha)}\right),\left(e^{-i(\pi / 2 \alpha)}, e^{-i(\pi / 2 \alpha)}\right)$. In fact, $1+x^{\alpha} y^{\alpha}=0$ implies $x^{\alpha} y^{\alpha}=-1$ and then $|x|=|y|=1$ and $\alpha(\arg x+\arg y)= \pm \pi$. In consequence,

$$
\begin{aligned}
& \arg x=\arg y=\frac{\pi}{2 \alpha} \text { or } \\
& \arg x=\arg y=-\frac{\pi}{2 \alpha} .
\end{aligned}
$$

For the denominator the argument is clear. Finally, $g_{\alpha}$ is continuous on the compact subset $\overline{S_{\alpha}(D) \times S_{\alpha}(D)}$ and does not vanish. In consequence, $g_{\alpha} \simeq 1$, on $\overline{S_{\alpha}(D) \times S_{\alpha}(D)}$.

Remark 23. Let $\alpha>1, \alpha \neq 1$. Then

$$
\frac{\delta_{1}(z) \delta_{2}(z) \delta_{3}(z)}{|z|\left|z-e^{i(\pi / 2 \alpha)}\right|\left|z-e^{-i(\pi / 2 \alpha)}\right|} \simeq \delta(z), \quad \text { on } S_{\alpha}(D)
$$

Proof. We have to discuss three cases.

Case 1. If $\delta_{1}(z) \leq \delta_{2}(z)$, then $\sin (\pi / 2 \alpha) \leq\left|z-e^{-i(\pi / 2 \alpha)}\right| \leq 2$, which implies that

$$
\begin{aligned}
\delta(z) & =\left(\delta_{1}(z) \wedge \delta_{3}(z)\right) \simeq \frac{\delta_{1}(z) \delta_{3}(z)}{\delta_{1}(z)+\delta_{3}(z)} \\
& \simeq \frac{\delta_{1}(z) \delta_{3}(z)}{\left|z-e^{i(\pi / 2 \alpha)}\right|} \simeq \frac{\delta_{1}(z) \delta_{2}(z) \delta_{3}(z)}{\left|z-e^{i(\pi / 2 \alpha)}\right||z|} \\
& \simeq \frac{\delta_{1}(z) \delta_{2}(z) \delta_{3}(z)}{\left|z-e^{i(\pi / 2 \alpha)}\right|\left|z-e^{-i(\pi / 2 \alpha)}\right||z|} .
\end{aligned}
$$
$\delta_{2}(z)$.

Using the same argument, we obtain the result if $\delta_{1}(z) \geq$

Case 2. If $\delta_{3}(z) \leq \delta_{1}(z)$, then $\delta_{1}(z) \simeq\left|z-e^{i(\pi / 2 \alpha)}\right|$ and $|z| \geq$ $1 / 2$, which implies

$$
\begin{aligned}
\delta(z) & =\left(\delta_{2}(z) \wedge \delta_{3}(z)\right) \simeq \frac{\delta_{2}(z) \delta_{3}(z)}{\delta_{2}(z)+\delta_{3}(z)} \\
& \simeq \frac{\delta_{2}(z) \delta_{3}(z)}{\left|z-e^{-i(\pi / 2 \alpha)}\right|} \\
& \simeq \frac{\delta_{1}(z) \delta_{2}(z) \delta_{3}(z)}{\left|z-e^{i(\pi / 2 \alpha)}\right|\left|z-e^{-i(\pi / 2 \alpha)}\right||z|}
\end{aligned}
$$

In the same way, we prove the result for $\delta_{1}(z) \leq \delta_{3}(z)$.

Case 3. If $\delta_{3}(z) \leq \delta_{2}(z)$, then $|z| \geq 1 / 2$, which implies

$$
\begin{aligned}
\delta(z) & =\left(\delta_{1}(z) \wedge \delta_{3}(z)\right) \simeq \frac{\delta_{1}(z) \delta_{3}(z)}{\left|z-e^{i(\pi / 2 \alpha)}\right|} \\
& \simeq \frac{\delta_{1}(z) \delta_{3}(z) \delta_{2}(z)}{\left|z-e^{i(\pi / 2 \alpha)}\right|\left|z-e^{-i(\pi / 2 \alpha)}\right|} \\
& \simeq \frac{\delta_{1}(z) \delta_{2}(z) \delta_{3}(z)}{\left|z-e^{i(\pi / 2 \alpha)}\right|\left|z-e^{-i(\pi / 2 \alpha)}\right||z|} .
\end{aligned}
$$

Remark 24. If $\alpha=1$, then for all $z \in S_{1}(D)$

$$
\delta_{1}(z) \delta_{2}(z) \delta_{3}(z)=|z| \mathfrak{R} e(z)(1-|z|) .
$$

Moreover, since $\left|1+z^{2}\right|=4(\Re e(z))^{2}+\left(1-|z|^{2}\right)^{2}$, then

$$
|z-i||z+i| \simeq(\mathfrak{R} e(z) \vee 1-|z|), \quad \text { on } S_{1}(D) \text {. }
$$


This implies that

$$
\begin{aligned}
& \delta_{1}(z) \delta_{2}(z) \delta_{3}(z)=|z| \mathfrak{R} e(z)(1-|z|) \\
& \quad \simeq|z|(\mathfrak{R} e(z) \wedge(1-|z|))(\mathfrak{R} e(z) \vee(1-|z|)) \\
& \quad \simeq|z| \delta(z)|z-i||z+i|, \quad \text { on } S_{1}(D) .
\end{aligned}
$$

Proof of Theorem 3. The Green function $G_{D}$ of $D$ is given, for all $x, y \in D$, by

$$
\begin{aligned}
G_{D}(x, y) & =\ln \left|\frac{1-x \bar{y}}{x-y}\right| \\
& =\frac{1}{2} \ln \left(1+\frac{\left(1-|x|^{2}\right)\left(1-|y|^{2}\right)}{|x-y|^{2}}\right) .
\end{aligned}
$$

Hence, the Green function of $S_{1}(D)$ is

$$
\begin{aligned}
G_{S_{1}(D)} & (x, y)=\ln \left(\left|\frac{1-x \bar{y}}{x-y}\right|\right)-\ln \left(\left|\frac{1+x y}{x+\bar{y}}\right|\right) \\
= & \ln \left|\frac{(1-x \bar{y})(x+\bar{y})}{(x-y)(1+x y)}\right| \\
= & \frac{1}{2} \ln \left(1+\frac{4 x_{1} y_{1}\left(1-|x|^{2}\right)\left(1-|y|^{2}\right)}{|(x-y)|^{2}|(1+x y)|^{2}}\right) .
\end{aligned}
$$

Since, the function $z \mapsto z^{\alpha}$ is a conformal mapping from $S_{\alpha}(D)$ onto $S_{1}(D)$, then for all $x, y \in S_{\alpha}(D)$

$$
\begin{aligned}
G_{S_{\alpha}(D)}(x, y)=G_{S_{1}(D)}\left(x^{\alpha}, y^{\alpha}\right)=\frac{1}{2} \ln (1 \\
\left.+\frac{4 \Re e\left(x^{\alpha}\right) \cdot \mathfrak{R} e\left(y^{\alpha}\right)\left(1-|x|^{2 \alpha}\right)\left(1-|y|^{2 \alpha}\right)}{\left|x^{\alpha}-y^{\alpha}\right|^{2}\left|1+x^{\alpha} y^{\alpha}\right|^{2}}\right) .
\end{aligned}
$$

On the other hand, if $t \in[0,1]$, then $1 \leq\left(1-t^{2 \alpha}\right) /(1-t) \leq 2 \alpha$. It follows that

$$
1-|z|^{2 \alpha} \simeq 1-|z|=\delta_{3}(z), \quad \forall z \in S_{\alpha}(D) .
$$

By Lemma 15, for all $z \in S_{\alpha}(D)$, we have $\mathfrak{R} e\left(z^{\alpha}\right)=$ $|z|^{\alpha} \cos (\alpha \arg z) \simeq|z|^{\alpha-2} \delta_{1}(z) \delta_{2}(z)$. By Lemma 14, $\left|x^{\alpha}-y^{\alpha}\right|^{2} \simeq(|x| \vee|y|)^{2 \alpha-2}|x-y|^{2}$. By Lemma 21, $\mid 1+$ $\left.x^{\alpha} y^{\alpha}\right|^{2} \simeq[x, y]_{\alpha, 2}[x, y]_{\alpha, 3}$. By Lemma 16, $[x, y]_{\alpha, 1} \simeq(|x| \vee$ $|y|)^{2}$. Hence, we obtain

$$
\begin{aligned}
& G_{S_{\alpha}(D)}(x, y) \simeq \ln (1 \\
& \left.+\frac{\delta_{1}(x) \delta_{1}(y) \delta_{2}(x) \delta_{2}(y) \delta_{3}(x) \delta_{3}(y)|x|^{\alpha-2}|y|^{\alpha-2}}{|x-y|^{2}(|x| \vee|y|)^{2 \alpha-2}[x, y]_{\alpha, 2}[x, y]_{\alpha, 3}}\right) \\
& \quad \simeq \ln (1 \\
& \left.+\frac{\delta_{1}(x) \delta_{1}(y) \delta_{2}(x) \delta_{2}(y) \delta_{3}(x) \delta_{3}(y)}{|x-y|^{2}[x, y]_{\alpha, 1}[x, y]_{\alpha, 2}[x, y]_{\alpha, 3}}\left(\frac{(|x| \wedge|y|)}{(|x| \vee|y|)}\right)^{\alpha-2}\right) .
\end{aligned}
$$

Relation (6) follows by using (87), Lemmas 14 and 15, and Proposition 22.

Proof of Corollary 4. The results follows by using (78) and (6) and the fact that $|x||y|=(|x| \vee|y|)(|x| \wedge|y|)$.

Remark 25. By using relations (83) and (86) of Proposition 22, we get

$$
\begin{aligned}
& G_{S_{1}(D)}(x, y) \simeq \ln \left(1+\left(\frac{|x-i| \wedge|y-i|}{|x-i| \vee|y-i|}\right)\right. \\
& \left.\cdot\left(\frac{|x+i| \wedge|y+i|}{|x+i| \vee|y+i|}\right) \frac{\delta(x) \delta(y)}{|x-y|^{2}}\right) \\
& \text { on } S_{1}(D) \times S_{1}(D) .
\end{aligned}
$$

\section{Estimates of the Green Function on $D \cap D(a, s)$}

We can see that

$$
\begin{aligned}
& \delta_{1}^{\star}(z)= \begin{cases}1-|z| & \text { if }|\arg z| \leq \theta_{0} \\
\left(\left|z-e^{i \theta_{0}}\right| \wedge\left|z-e^{-i \theta_{0}}\right|\right) & \text { if }|\arg z| \geq \theta_{0},\end{cases} \\
& \delta_{2}^{\star}(z) \\
& \quad= \begin{cases}s-|z-a| & \text { if }|\arg (z-a)| \geq \theta_{1} \\
\left(\left|z-e^{i \theta_{1}}\right| \wedge\left|z-e^{-i \theta_{1}}\right|\right) & \text { if }|\arg (z-a)| \leq \theta_{1} .\end{cases}
\end{aligned}
$$

Lemma 26. Let $a_{1}, a_{2}, b_{1}, b_{2}, k_{1}, k_{2}>0$ such that $k_{1} \leq a_{1}+a_{2} \leq$ $k_{2}$ and $k_{1} \leq b_{1}+b_{2} \leq k_{2}$. Then,

$$
\begin{aligned}
& \text { (1) }\left(a_{1} b_{2} \vee a_{2} b_{1}\right) \simeq\left(a_{1} \vee b_{1}\right)\left(a_{2} \vee b_{2}\right), \\
& \text { (2) }\left(a_{1} b_{2} \wedge a_{2} b_{1}\right) \simeq\left(a_{1} \wedge b_{1}\right)\left(a_{2} \wedge b_{2}\right) .
\end{aligned}
$$

Proof. (1) It is clear that $\left(a_{1} b_{2} \vee a_{2} b_{1}\right) \leq\left(a_{1} \vee b_{1}\right)\left(a_{2} \vee b_{2}\right)$. Inversely, we must prove that $\left(a_{1} b_{2}+a_{2} b_{1}\right) /\left(\left(a_{1}+b_{1}\right)\left(a_{2}+b_{2}\right)\right)$ is lower bounded.

(a) If $a_{1} \leq a_{2}, b_{1} \leq b_{2}$ (or $a_{2} \leq a_{1}, b_{2} \leq b_{1}$ ), then $a_{2} \geq k_{1} / 2$, $b_{2} \geq k_{1} / 2$. This implies

$$
\frac{a_{1} b_{2}+a_{2} b_{1}}{\left(a_{1}+b_{1}\right)\left(a_{2}+b_{2}\right)} \geq \frac{k_{1}}{2 k_{2}} .
$$

(b) If $a_{1} \leq a_{2}, b_{2} \leq b_{1}$ (or $a_{2} \leq a_{1}, b_{1} \leq b_{2}$ ), then $a_{2} \geq$ $k_{1} / 2, b_{1} \geq k_{2} / 2$. This implies $k_{1} / 2 \leq a_{1}+b_{1} \leq 2 k_{2}$, $k_{1} / 2 \leq a_{2}+b_{2} \leq 2 k_{2}, k_{1}^{2} / 4 \leq a_{2} b_{1}$ and so

$$
\frac{a_{1} b_{2}+a_{2} b_{1}}{\left(a_{1}+b_{1}\right)\left(a_{2}+b_{2}\right)} \geq \frac{k_{1}^{2}}{16 k_{2}^{2}} \text {. }
$$

For (2), we use the fact that $(a \wedge b)=a b /(a \vee b)$.

Proof of Theorem 5. The conformal mapping $\phi(z)=$ $e^{i\left(\left(\pi-\theta_{0}-\theta_{1}\right) / 2\right)}\left(\left(e^{i \theta_{0}}-z\right) /\left(z-e^{-i \theta_{0}}\right)\right)$ is a one to one application 
from $D \cap D(a, r)$ onto the sector $S_{\alpha}=\{z \in \mathbb{C}:|\arg z|<$ $\left.\left(\pi+\theta_{0}-\theta_{1}\right) / 2=\pi / 2 \alpha\right\}$, where $\alpha=\pi /\left(\pi+\theta_{0}-\theta_{1}\right)>1$. More precisely,

$$
\begin{aligned}
& \phi\left(C_{1}\right)=\left\{t e^{i(\pi / 2 \alpha)}, t \geq 0\right\}, \\
& \phi\left(C_{2}\right)=\left\{t e^{-i(\pi / 2 \alpha)}, t \geq 0\right\} .
\end{aligned}
$$

The Green function of $D^{\prime}=D \cap D(a, r)$ is given for $x, y \in D^{\prime}$ by

$$
G_{D^{\prime}}(x, y)=\ln \left|\frac{\phi^{\alpha}(x)+\overline{\phi^{\alpha}(y)}}{\phi^{\alpha}(x)-\phi^{\alpha}(y)}\right|
$$

From (4), the Green function estimates on $S_{\alpha}$, we have

$$
G_{D^{\prime}}(x, y) \simeq \ln \left(1+\frac{\delta_{1}(\phi(x)) \delta_{1}(\phi(y)) \delta_{2}(\phi(x)) \delta_{2}(\phi(y))}{|\phi(x)-\phi(y)|^{2}(|\phi(x)| \vee|\phi(y)|)^{2}}\left(\frac{(|\phi(x)| \wedge|\phi(y)|)}{(|\phi(x)| \vee|\phi(y)|)}\right)^{\alpha-2}\right)
$$

But,

$$
|\phi(x)-\phi(y)|^{2}=\frac{|x-y|^{2}}{\left|x-e^{-i \theta_{0}}\right|^{2}\left|y-e^{-i \theta_{0}}\right|^{2}}
$$

Besides, by Lemma 26,

$$
\begin{aligned}
& (|\phi(x)| \vee|\phi(y)|)=\left(\left|\frac{x-e^{i \theta_{0}}}{x-e^{-i \theta_{0}}}\right| \vee\left|\frac{y-e^{i \theta_{0}}}{y-e^{-i \theta_{0}}}\right|\right) \\
& =\frac{1}{\left|x-e^{-i \theta_{0}}\right|\left|y-e^{-i \theta_{0}}\right|}\left(\left|x-e^{i \theta_{0}}\right|\left|y-e^{-i \theta_{0}}\right|\right. \\
& \left.\vee\left|y-e^{i \theta_{0}}\right|\left|x-e^{-i \theta_{0}}\right|\right) \\
& \simeq \frac{1}{\left|x-e^{-i \theta_{0}}\right| \mid y-e^{-i \theta_{0} \mid}}\left(\left|x-e^{i \theta_{0}}\right| \vee\left|y-e^{i \theta_{0}}\right|\right) \\
& \cdot\left(\left|x-e^{-i \theta_{0}}\right| \vee\left|y-e^{-i \theta_{0}}\right|\right) .
\end{aligned}
$$

Moreover, we can verify that, for $i=1, i=2: \delta_{i}(\phi(z)) \simeq$ $\delta_{i}^{*}(z) /\left|z-e^{-i \theta_{0}}\right|^{2}$. Thus the results follow.

Proof of Corollary 6. From the property of Lemma 17, we deduce for all $z \in D \cap D(a, r)$

$$
\left(\delta_{1}^{*}(z) \vee \delta_{2}^{*}(z)\right) \simeq\left|z-e^{i \theta_{0}}\right|\left|z-e^{-i \theta_{0}}\right| .
$$

For $z \in D \cap \mathscr{D}(a, r)$, we have

$$
\begin{aligned}
\left(\delta_{1}(\phi(z)) \vee \delta_{2}(\phi(z))\right) & \simeq|\phi(z)| \\
\delta_{i}(\phi(z)) & \simeq \frac{\delta_{i}^{*}(z)}{\left|z-e^{-i \theta_{0}}\right|} .
\end{aligned}
$$

Now, we see that, for $z \in D \cap D(a, r)$,

$$
\begin{aligned}
\delta^{*}(z) & =\left(\delta_{1}^{*}(z) \wedge \delta_{2}^{*}(z)\right) \simeq \frac{\delta_{1}^{*}(z) \delta_{2}^{*}(z)}{\delta_{1}^{*}(z)+\delta_{2}^{*}(z)} \\
& \simeq \frac{\delta_{1}^{*}(z) \delta_{2}^{*}(z)}{\left|z-e^{i \theta_{0}}\right|\left|z-e^{-i \theta_{0}}\right|} .
\end{aligned}
$$

Thus the results follow.

\section{Estimates of the Green Function on $D \cup D(a, s)$}

The conformal mapping

$$
\phi(z)=e^{i\left(\left(\pi-\theta_{0}-\theta_{1}\right) / 2\right)} \frac{e^{i \theta_{0}}-z}{z-e^{-i \theta_{0}}}
$$

is a one to one application from $D \cup D(a, s)$ onto $S_{\beta}=\{z \in$ $\mathbb{C},|\arg z| \leq \pi / 2 \beta\}$, where $\beta=\alpha /(2 \alpha-1)$. The Green function is given for $x, y \in D^{\prime \prime}=D \cup D(a, s)$ by

$$
G_{D^{\prime \prime}}(x, y)=\ln \left|\frac{\phi^{\beta}(x)+\overline{\phi^{\beta}(y)}}{\phi^{\beta}(x)-\phi^{\beta}(y)}\right| .
$$

The same analysis leads to analogous estimates. The boundary of $D \cup D(a, s)$ is formed by the two arcs of circles

$$
\begin{aligned}
& \mathscr{C}_{1}^{\prime}=\left\{z \in \mathbb{C},|z|=1,|\arg z| \geq \theta_{0}\right\}, \\
& \mathscr{C}_{2}^{\prime}=\left\{z \in \mathbb{C},|z-a|=s,|\arg z| \leq \theta_{1}\right\} .
\end{aligned}
$$

We denote by $\delta_{i}^{\prime}(z)$ the distance of $z \in D \cup D(a, s)$ to the boundary $\mathscr{C}_{i}^{\prime}, i \in\{1,2\}$. For $z \in D \cup D(a, s)$

$$
\begin{aligned}
& \delta_{1}^{\prime}(z)= \begin{cases}|1-| z|| & \text { if }|\arg z| \geq \theta_{0} \\
\left(\left|z-e^{i \theta_{0}}\right| \wedge\left|z-e^{-i \theta_{0}}\right|\right) & \text { if }|\arg z| \leq \theta_{0}\end{cases} \\
& \delta_{2}^{\prime}(z) \\
& = \begin{cases}|s-| z-a|| & \text { if }|\arg (z-a)| \leq \theta_{1} \\
\left(\left|z-e^{i \theta_{1}}\right| \wedge\left|z-e^{-i \theta_{1}}\right|\right) & \text { if }|\arg (z-a)| \geq \theta_{1}\end{cases} \\
& \delta(z)=\left(\delta_{1}^{\prime}(z) \wedge \delta_{2}^{\prime}(z)\right) \simeq \frac{\delta_{1}^{\prime}(z) \delta_{2}^{\prime}(z)}{\delta_{1}^{\prime}(z)+\delta_{2}^{\prime}(z)} \\
& \simeq \frac{\delta_{1}^{\prime}(z) \delta_{2}^{\prime}(z)}{\left|z-e^{i \theta_{0}}\right|\left|z-e^{-i \theta_{0}}\right|} .
\end{aligned}
$$

Thus, we obtain Theorem 7. 


\section{Estimates of the Green Function on}

$D \backslash D(a, s)$

The conformal mapping

$$
i \phi(z)=i e^{i\left(\left(\pi-\theta_{0}-\theta_{1}\right) / 2\right)} \frac{e^{i \theta_{0}}-z}{z-e^{-i \theta_{0}}}
$$

is a one to one application from $D_{0}=D \backslash D(a, r)$ onto the sector $S_{\gamma}=\{z \in \mathbb{C},|\arg z|<((\alpha-1) / \alpha) \pi=\pi / 2 \gamma\}$, where $\gamma=\alpha /(\alpha-1)>1$. The Green function is given for $x, y \in$ $D_{0}=D \backslash D(a, r)$ by

$$
G_{D_{0}}(x, y)=\ln \left|\frac{(i \phi(x))^{\gamma}+\overline{(i \phi(y))^{\gamma}}}{(i \phi(x))^{\gamma}-(i \phi(y))^{\gamma}}\right| .
$$

The boundary of $D \backslash D(a, r)$ is formed by two arcs of circles

$$
\begin{aligned}
& \mathscr{C}_{1}^{\prime \prime}=\left\{z \in \mathbb{C},|z|=1,|\arg z| \geq \theta_{0}\right\}, \\
& \mathscr{C}_{2}^{\prime \prime}=\left\{z \in \mathbb{C},|z-a|=s,|\arg (z-a)| \geq \theta_{1}\right\} .
\end{aligned}
$$

We denote by $\delta_{i}^{\prime \prime}(z)$ the distance of $z \in D \backslash D(a, s)$ to the boundary $C_{i}^{\prime \prime}, i=1,2$. Consider

$$
\begin{aligned}
& \delta_{1}^{\prime \prime}(z)= \begin{cases}1-|z| & \text { if }|\arg z| \geq \theta_{0} \\
\left(\left|z-e^{i \theta_{0}}\right| \wedge\left|z-e^{-i \theta_{0}}\right|\right) & \text { if }|\arg z| \leq \theta_{0}\end{cases} \\
& \delta_{2}^{\prime \prime}(z) \\
& = \begin{cases}|z-a|-s & \text { if }|\arg (z-a)| \geq \theta_{1} \\
\left(\left|z-e^{i \theta_{1}}\right| \wedge\left|z-e^{-i \theta_{1}}\right|\right) & \text { if }|\arg (z-a)| \leq \theta_{1} .\end{cases}
\end{aligned}
$$

We find using the same analysis Theorem 8 .

\section{Estimates of the Green Function on $D \cap\{z \in$}

$$
\left.\mathbb{C}, \mathfrak{R} e(z)>\cos \theta_{0}\right\}, 0<\theta_{0}<\pi / 2
$$

The conformal mapping

$$
\psi(z)=e^{-i\left(\theta_{0} / 2\right)} \frac{e^{i \theta_{0}}-z}{z-e^{-i \theta_{0}}}
$$

is a one to one application from this domain onto the sector $S_{\nu}=\left\{z \in \mathbb{C},|\arg z|<\theta_{0} / 2=\pi / 2 \nu\right\}$, where $\nu=\pi / \theta_{0} \geq 2$. The Green function is given for $x, y \in D_{1}=D \cap\{z \in \mathbb{C}, \mathfrak{R} e(z)>$ $\left.\cos \theta_{0}\right\}$ by

$$
G_{D_{1}}(x, y)=\ln \left|\frac{\psi^{\nu}(x)+\overline{\psi^{\nu}(y)}}{\psi^{\nu}(x)-\psi^{\nu}(y)}\right| .
$$

The boundary of this domain is formed by the arc of the unit circle $\left\{z \in \mathbb{C},|z|=1,|\arg z|<\theta_{0}\right\}$ and the segment $\left[e^{-i \theta_{0}}, e^{i \theta_{0}}\right]$. For $z \in D \cap\left\{z \in \mathbb{C}, \mathfrak{R} e(z)>\cos \theta_{0}\right\}$, let us put

$$
\begin{aligned}
& \delta_{1}^{\prime \prime \prime}(z)=1-|z|, \\
& \delta_{2}^{\prime \prime \prime}(z)=\mathfrak{R} e(z)-\cos \theta_{0} .
\end{aligned}
$$

We find using the same analysis Theorem 9.

\section{Conflict of Interests}

The authors declare that there is no conflict of interests regarding the publication of this paper.

\section{References}

[1] Z. Zhao, "Green functions and conditioned gauge theorem for a 2-dimensional domain," in Seminar on Stochastic Processes, 1987, vol. 15 of Progress in Probability and Statistics, pp. 283-294, Birkhäuser, Boston, Mass, USA, 1988.

[2] M. Selmi, "Inequalities for green functions in a dini-jordan domain in $\mathbb{R}^{2}$," Potential Analysis, vol. 13, no. 1, pp. 81-102, 2000.

[3] G. Sweers, "Guido Hopf's lemma and two-dimensional domains with corners. Dedicated to the memory of Pierre Grisvard," Rendiconti dell'Istituto di Matematica dell'Università di Trieste, vol. 28, supplement, pp. 383-419, 1996-1997.

[4] L. V. Ahlfors, Complex Analysis: An Introduction to the Theory of Analytic Functions of One Complex Variable, McGraw-Hill, 2nd edition, 1966. 


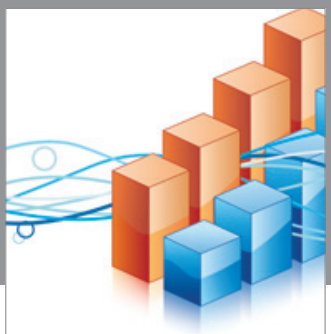

Advances in

Operations Research

vatem alat4

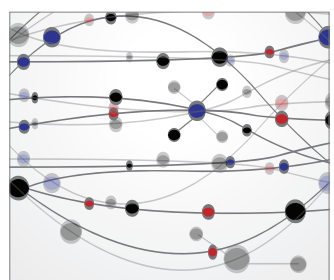

\section{The Scientific} World Journal
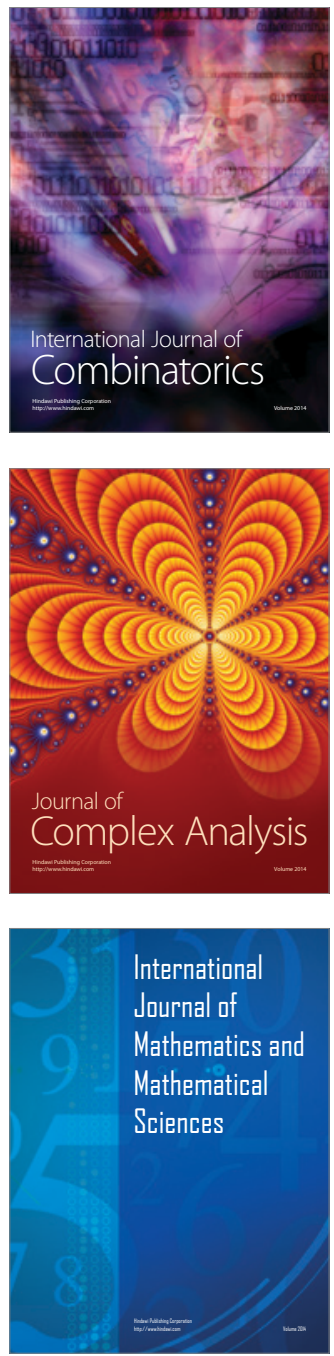
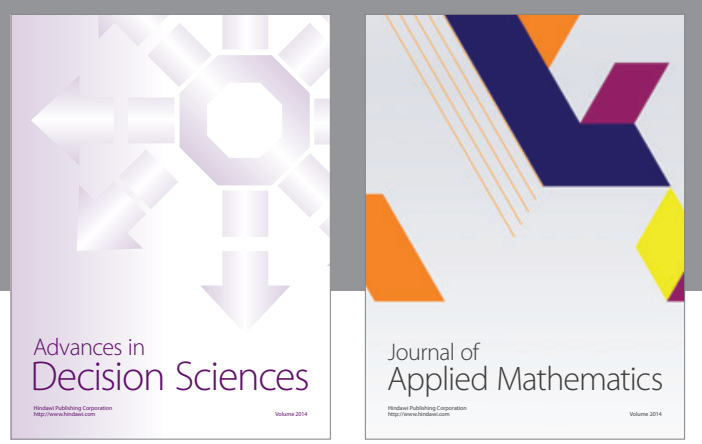

Algebra

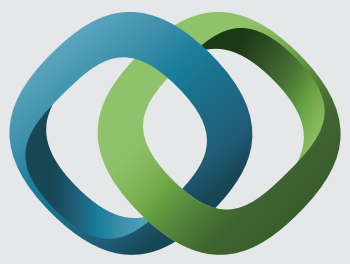

\section{Hindawi}

Submit your manuscripts at

http://www.hindawi.com
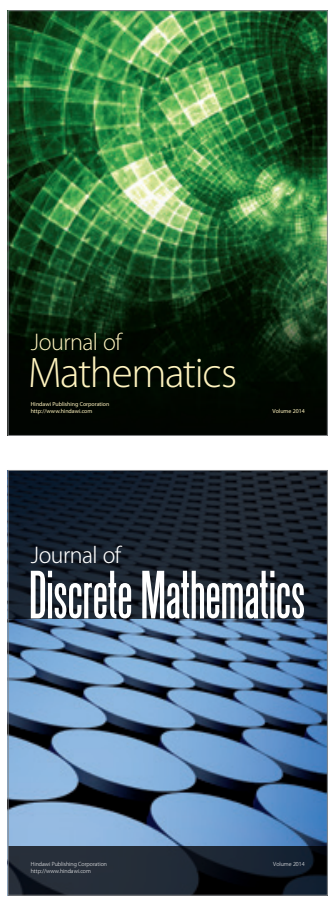

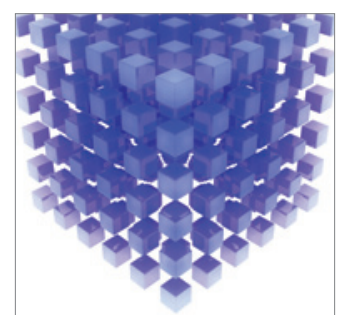

Mathematical Problems in Engineering
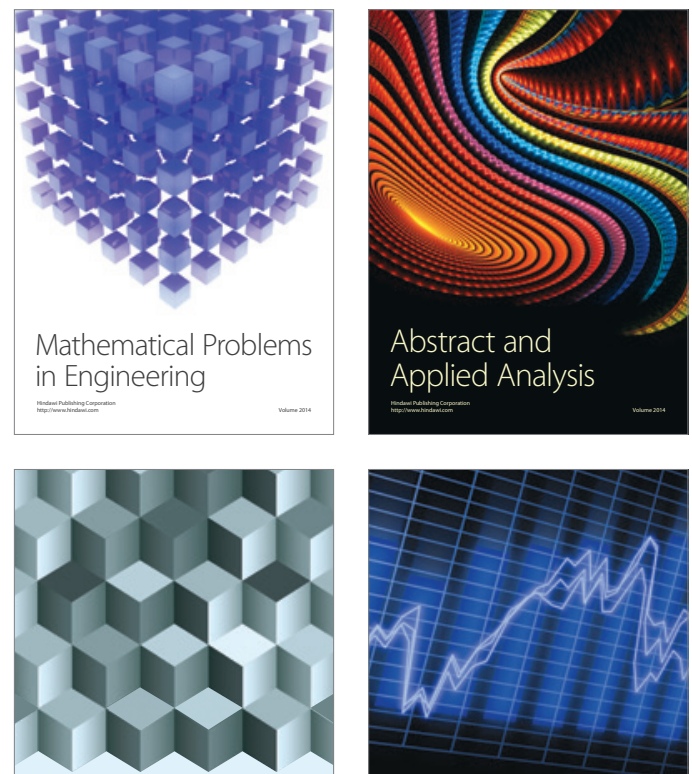

Journal of

Function Spaces

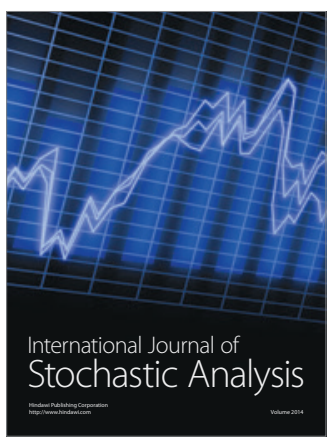

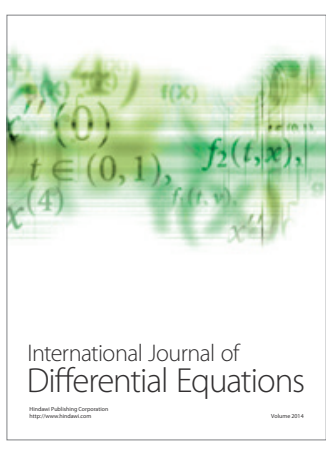
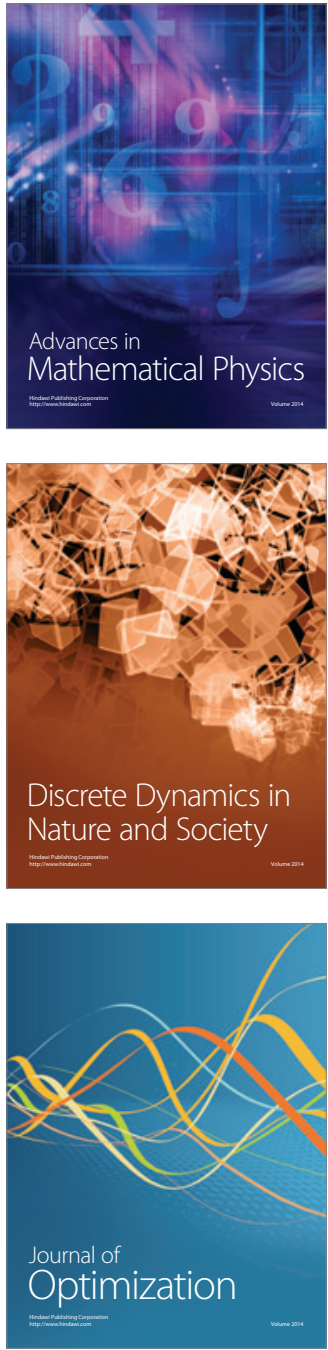